\title{
时空信息联合嵌入的端到端三维模型草图检索
}

\author{
白静 ${ }^{1,2}$, 周文惠 ${ }^{1)}$, 拖继文 ${ }^{1)}$, 秦飞巍 ${ }^{3)}$ \\ 1) (北方民族大学计算机科学与工程学院 银川 750021) \\ 2) (宁夏智能信息与大数据处理重点实验室 银川 750021) \\ 3) (杭州电子科技大学计算机学院 杭州 310018) \\ (baijing_nun@163.com)
}

\begin{abstract}
摘 要: 现有的基于草图的三维模型检索工作往往将数据视为静态输人, 并使用卷积神经网络进行特征提取, 忽略 了数据的动态属性，造成了有益信息的部分丢失，进而影响了以此为基础的检索效果. 为解决这一问题，提出一种基 于时空信息联合嵌人的端到端三维模型草图检索算法. 首先, 将草图表征为动态绘制序列, 体现其绘制过程中所包 含的时序信息; 将三维模型表征为多视图序列，体现视图间的位置关联; 然后，构建包含静态空间特征提取和动态 时序特征提取的端到端双流网络, 结合三元中心度量学习建立跨域数据的联合时空特征嵌人, 充分捕捉草图和三维 模型所包含的静态、动态特征, 缩小跨域数据的差异性, 提高检索的准确率; 最后, 在标准公开数据集 SHREC2013 和 SHREC2014 上进行实验, 与现有工作相比获得了较高的准确率, 验证了所提算法的可行性及有效性.
\end{abstract}

关键词：三维模型检索；基于草图的检索；双流网络；时空特征；度量学习；深度学习

中图法分类号: TP391.41

DOI: $10.3724 /$ SP.J.1089.2021.18574

\section{End-to-End Sketch-3D Model Retrieval with Spatiotemporal Information Joint Embedding}

\author{
Bai Jing ${ }^{1,2)}$, Zhou Wenhui ${ }^{1)}$, Tuo Jiwen ${ }^{1)}$, and Qin Feiwei ${ }^{3)}$ \\ 1) (School of Computer Science and Engineering, North Minzu University, Yinchuan 750021) \\ 2) (Ningxia Province Key Laboratory of Intelligent Information and Data Processing, Yinchuan 750021) \\ 3) (School of Computer Science and Technology, Hangzhou Dianzi University, Hangzhou 310018)
}

\begin{abstract}
The existing sketch-based 3D model retrieval methods often regard data as static input, and utilize convolutional neural network to extract features, which ignore the dynamic attributes of input data, resulting in partial loss of useful information and not ideal retrieval effect. In order to solve this problem, a spatiotemporal information joint embedding-based end-to-end sketch-3D model retrieval method is proposed. Firstly, the sketch is represented as a dynamic drawing sequence, which reflects the temporal information contained in the drawing process; meanwhile, the 3D model is represented as a multi-view sequence to reflect the position relationship between views. Secondly, an end-to-end dual-stream network including static spatial feature extraction and dynamic time series feature extraction is constructed. Combined with triplet central metric learning, a joint spatiotemporal feature embedding of cross domain data is established to fully capture the static and dynamic features contained in sketches and 3D models, and reduce the difference between cross domain. Finally, experiments are carried out on the standard public data sets SHREC2013 and SHREC2014. Compared with the ex-
\end{abstract}

收稿日期：2020-08-13; 修回日期：2020-10-01. 基金项目：国家自然科学基金(61762003，61972121); 中国科学院“西部之光”人 才培养引进计划; 宁夏高等学校一流学科建设(NXYLXK2017A07); 宁夏优秀人才支持计划; 北方民族大学“计算机视觉和虚拟现实” 创新团队项目. 白静(1982-), 女, 博士, 教授, 硕士生导师, CCF 高级会员, 主要研究方向为 CAD\&CD、机器学习; 周文惠(1996一), 女, 硕士研究生, 主要研究方向为机器学习; 拖继文(1995一), 男, 硕士研究生, 主要研究方向为机器学习; 秦飞巍(1985一), 男, 博 士, 副教授, CCF 会员, 主要研究方向为 CAD\&CG、机器学习. 
isting work, the accuracy rate is higher, which verifies the feasibility and effectiveness of the proposed algorithm.

Key words: 3D model retrieval; sketch-based retrieval; dual-stream network; spatiotemporal feature; metric learning; deep learning

随着计算机辅助设计与计算机视觉的快速发 展, 三维物体作为一种重要的数据类型成为继声 音、图像与视频之后信息的主要载体之一, 并在工 业制造、虚拟现实和增强现实等领域有着广泛的应 用. 如何有效地识别和检索三维模型是诸多应用 的研究基础, 已成为相关学者关注的课题, 出现了 一系列研究成果 ${ }^{[1-2]}$.

手绘草图易于构建，非常直观，且不受地域、 专业、年龄等外在因素影响, 在人类历史中一直被 当作一种非常有效的交流手段. 近年来，随着便携 式触屏设备的普及, 手绘草图数据变得易于获取, 基于手绘草图的三维模型检索成为新的研究方向. 然而，三维模型和草图之间存在着巨大的域间差 异性: 三维模型数据是现实世界的客观表征或虚 拟世界的数字化模型, 具有表征准确、具体, 数据 维度高、非结构化等特性; 而草图是用户的一种主 观表达, 往往由表征物体全局属性的简单线条组 成, 强调整体结构和突出特点, 具有稀疏性和全局 性等特性. 因而, 基于手绘草图的三维模型检索仍 然非常困难.

传统的基于手绘草图的三维模型检索算法往 往利用剪影图和外轮廓线图将三维模型表征为二 维线条图, 然后利用人工设计的特征编码算法, 如 弥散张量 ${ }^{[3]}$ 、费舍编码 ${ }^{[4]}$ 及基于局部特征的多尺度 描述 ${ }^{[5]}$ 等表征三维模型和草图, 最后在特征空间利 用某种相似评价模型完成距离度量, 实现草图到 三维模型的检索. 由于三维模型和草图域间差异 巨大, 三维模型又非常复杂, 这类基于人工设计特 征的算法往往无法客观地捕捉三维模型和草图的 本质特征, 因而整体检索准确率偏低.

与传统算法相比, 深度学习可以让机器自动 学习被表征对象的特征描述符, 广泛地应用于计 算机视觉领域. 相应地, 在基于草图的三维模型检 索中也出现了一系列优秀的工作 ${ }^{[6-13]}$, 并取得了不 错的检索效果. 但不可忽视的是, 目前的三维模型 的草图检索仍然面临一些难题. (1) 与图像不同, 手绘草图是一个动态序列, 现有算法往往利用卷 积神经网络 (convolutional neural networks, CNN)针 对草图绘制的最终结果提取描述符, 忽略了手绘 草图包含的丰富的时序信息 ${ }^{[14]}$. (2) 三维模型具有
复杂性, 现有算法往往将三维模型表征为多个独 立视图, 通过多通道完成特征提取, 忽略了视图之 间的关联关系. 以上 2 个问题相互作用, 严重影响 了三维模型草图检索的整体性能.

针对以上问题，本文提出一种基于时空信息 联合嵌人的端到端三维模型草图检索算法.

(1) 将草图和三维模型表征为图像序列, 并引 人残差网络和长短期记忆网络 (long short-term memory, LSTM), 建立时空特征提取双流网络, 同 时捕捉草图和三维模型的静态空间特征和动态时 序特征, 更加全面、准确地刻画被表征对象.

(2) 提出一种基于时空信息联合嵌人的端到 端三维模型草图检索框架, 结合具有时空特征提 取能力的双流网络和三元度量学习建立跨域数据 的联合时空特征嵌人, 缩小跨域数据的差异性, 提 高检索准确率.

在公共数据集 SHREC2013 和 SHREC2014 上 实验验证了本文算法的有效性.

\section{1 相关工作}

通常, 基于深度学习的三维模型草图检索包 括跨域数据的初始表征、特征嵌人和相似度计算. 相似度计算和传统算法类似, 本文不再赘述. 本节 将围绕跨域数据的初始表征和特征嵌人, 简单梳 理基于深度学习的三维模型草图检索算法.

\section{1 跨域数据的初始表征}

在三维模型草图检索中, 研究者往往将草图 看做一幅静态图像, 使用经典的 CNN 对草图进行 特征表示, 并提取草图初始特征, 如 AlexNet ${ }^{[6]}$ 和 $\mathrm{ResNet}^{[7]}$. 由于 $\mathrm{CNN}$ 本是针对自然图像设计的, 旨在获取图像中有判别性的纹理特征; 而手绘草 图较为抽象, 仅由简单线条构成, 缺乏颜色和纹理 信息, 因此仅使用 CNN 对草图进行特征提取效果 还不够理想. 此外, 草图的绘制是一个动态过程, 仅使用 CNN 的算法往往忽略了草图绘制过程中包 含的时序信息, 造成有益信息的损失, 这也进一步 影响了草图特征提取的效果.

考虑三维模型的非结构化特性, 在三维模型 草图检索中, 研究者往往将三维模型转换为一个 
或一组代表性视图, 以降低跨域匹配的难度. 刘玉 杰等 ${ }^{[6]}$ 提出基于视图信息熵选择一张代表性视图 表征三维模型，在保证检索效果的同时，简化了网 络结构. 由于草图并不对应三维模型某一个固定 视角的视图，因而最佳视图的构建是极其困难的. 为此, Wang 等 ${ }^{[8]}$ 提出针对坚直摆放的三维模型随 机选择 2 个大小间隔超过 $45^{\circ}$ 的投影视图表征三维 模型; Xie 等 ${ }^{[9]}$ 提出使用多张视图表征三维模型, 并最终采用瓦瑟斯坦重心融合多视图特征. 以上 算法是三维模型的多视图表征的有益尝试，取得 了良好效果. 然而，三维模型的多个视图之间是存 在位置次序的，而现有算法独立看待每个视图，忽 略了这种相关性，势必造成一定程度的信息损失.

本文针对草图和三维模型表征中存在的问题, 提出一种将 CNN 和循环神经网络(recurrent neural networks，RNN)相结合的草图-三维模型检索算法, 同时捕捉草图的空间信息和绘制过程所包含的时 序信息，以及三维模型多视图的空间信息和位次 信息，以提高跨域数据表征的准确性.

\section{2 跨域数据的特征嵌入}

部分基于草图的三维模型检索算法在完成三 维模型和草图的初始特征提取后，直接进行相似 评价. 例如, 刘玉杰等 ${ }^{[6]}$ 在利用 $\mathrm{CNN}$ 提取草图和 三维模型的特征后，便采用最小距离法进行相似 评价. 然而由于没有充分考虑跨域数据之间的差 异性，这类算法的检索效果不够理想.

更多的算法使用度量学习将草图和三维模型 的初始特征嵌人到一个公共空间中，使特征空间 中同类数据(同域和跨域)的距离更近，异类数据的 距离更远. 例如, Wang 等 ${ }^{[8]}$ 使用二元度量学习网络 Siamese，迫使跨域同类数据之间的距离足够近，完 成跨域数据的表征和嵌人. 考虑跨域数据特征的相 关性，Dai 等 ${ }^{[10]}$ 提出基于鉴别损失(discriminative loss)和相关损失 (correlation loss)的跨域特征嵌人 的深度度量相关算法(deep correlated metric learning, DCML), 有效地提高了域内不同类别数据特 征的鉴别性和域间同类别数据特征的相关性. 文 献[12-13]同时考虑同类数据和异类数据之间的关 系，提出基于三元度量学习三元损失(triplet loss) 的跨域数据嵌人，取得了很好的效果. 然而，基于 triplet loss 的算法需要考虑每一对正负样本组合, 网络训练费时且困难. 本文引人 triplet-center $10 \mathrm{~s} \mathrm{~s}^{[15]}$, 在减少计算复杂度的同时, 确保类内距离 最小化，类间距离最大化

\section{2 本文算法}

如图 1 所示, 本文提出了一种基于时空信息联 合嵌人的端到端三维模型草图检索框架. 在学习 阶段, 模拟人脑对视频特征的识别过程, 分别将草 图和三维模型表征为草图绘制序列和多视图序列; 然后利用时空特征提取网络提取静态空间特征和 动态时序特征, 并引人度量学习完成时空特征的 联合嵌人. 在检索阶段，输人草图提取其时空特 征，然后基于相似评价在联合时空特征分布空间 中搜索并返回相似度最高的 $k$ 个结果, 完成草图三维模型检索.

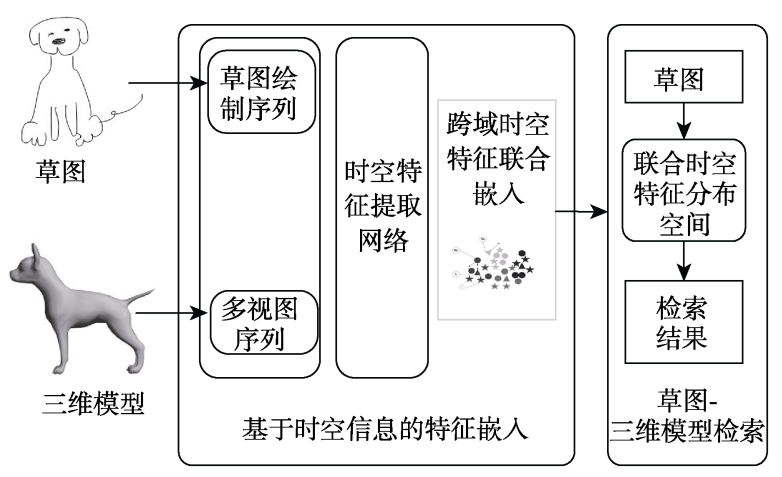

图 1 基于时空信息联合嵌人的三维模型草图检索框架

\section{1 图像序列的构建}

\section{1 .1 草图绘制序列的构建}

2012 年, Eitz 等 ${ }^{[14]}$ 对 “人类如何绘制草图”这一 问题进行了深人研究, 发现人类绘制草图的顺序 出奇的一致，即草图的绘制顺序蕴含着某种潜在 语义. 基于这一发现, 在草图识别中研究者也提出 了一些捕捉草图时序信息的算法, 并取得了较好 的效果 ${ }^{[16-19]}$. 然而，在三维模型草图检索中，同时 存在三维模型和草图 2 种跨域数据, 如何有效地提 取并应用草图的时序信息仍然是一个难题，迄今 为止尚无相关研究.

给定一张草图 $s$, 为充分捕捉其绘制过程中 所包含的时序信息, 本文将其表征为 $m$ 张笔画数 不断累加的草图绘制序列, 有

$$
\begin{gathered}
\partial(s)=\left(s_{1}, \cdots, s_{i}, s_{i+1}, \cdots, s_{m}\right), s_{m}=s, \\
\forall 1 \leqslant i<m, s_{i} \subseteq s_{i+1},\left|s_{i}\right| \leqslant\left|s_{i+1}\right| .
\end{gathered}
$$

其中, $\left|s_{i}\right|$ 表示子图像序列 $s_{i}$ 中所包含的笔画数目. 为确保草图绘制序列中，每一幅子图像都包含足 够的绘制信息. 更进一步, 本文要求子图像序列间 笔画的递增数目相当, 即要求

$$
\forall 1 \leqslant i \leqslant j<m \text {, 有 }\left|s_{j+1}\right|-\left|s_{j}\right|=\left|s_{i+1}\right|-\left|s_{i}\right| \text {. }
$$


针对三维模型草图检索数据集 SHREC2013 $3^{[20-21]}$ 和 SHREC2014 ${ }^{[22-23]}$, TU-Berlin ${ }^{[14]}$ 提供了三维模型 所包含的草图数据. 具体地, Eitz 等 ${ }^{[14]}$ 在 Amazon Mechanical Turk 平台上付费邀请了 1350 个人对各 类草图进行绘制, 并保留其绘制顺序, 形成了数据 量庞大且包含不同绘制人绘制习惯的大规模草图 数据集 TU-Berlin. 因此，本文针对草图绘制序列 的构建需求, 基于 TU-Berlin 数据集, 以包含用户 绘制笔画顺序信息的 svg 格式草图为输人, 构建草 图绘制序列提取算法.

输人. 草图数据 $s$, 子图序列个数 $m$.

输出. 草图绘制序列 $\partial(s)$.

Step1. 获取草图数据 $s$ 的笔画数 $n$.

Step2. 构建各个子图像:

$$
\begin{aligned}
& a=n / m ; b=n \% m ; \\
& \text { for }(i=1, j=1 ; i \leqslant b ; i++)\{
\end{aligned}
$$

子图 $i$ 的笔画为第 1 画 第 $j+a$ 画;

$j=j+a+1 ;\}$

for $(; i \leqslant m ; i++)\{$

子图 $i$ 的笔画为第 1 画 第 $j+a-1$ 画;

$j=j+a$;

Step 3. 输出子图序列 $\partial(s)$

当草图数据笔画数 $n$ 不能被 $m$ 整除时, 无法
严格保证各个子图中累加的笔画数完全一致. 此 时, 若 $n$ 对 $m$ 求余所得余数为 $b$, 则为第 1 张至第 $b$ 张子图每张增加 1 画, 即它们所对应的笔画数为 $a+1$; 其余子图笔画数为 $a$.

当笔画数 $n<m$ 时, 确保第 1 张至第 $b$ 张子图 每张递增 1 画，其余子图和第 $b$ 张子图保持一致 即可.

在以上 2 种情况下，无法严格保证每张子图累 加笔画数一致, 但是可以保证数目变化最多为 1 , 在一定程度上确保了各个子图包含信息的一致性.

图 2 随机选取了 3 张草图, 并给出了当子图数 目 $m$ 取 2,3,4, 即草图绘制过程分别为 2 步、3 步、 4 步时所对应的草图序列. 由图 2 可见，当子图数 目取值为 2 时，时域信息包含较少，仅体现了 1 组 由部分到全局的时序变化; 当子图数目为 3 和 4 时, 草图绘制序列包含了 2 3 个时序变化, 能够较好地 体现动态绘制过程. 同时，观察草图绘制序列会发 现: 在绘制草图时，用户通常会首先绘制体现草图 整体结构的外轮廓线条，然后绘制体现局部结构 的其他线条. 这也再次说明了草图绘制的动态过 程蕴含着丰富的时序信息，对这些信息的表征将 有助于草图的表征和识别.

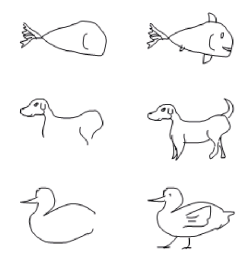

a. 草图绘制(2步)

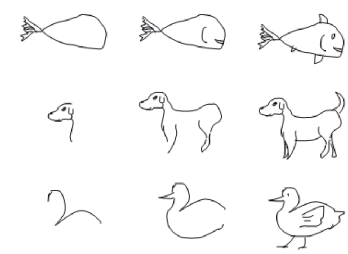

b. 草图绘制(3步)

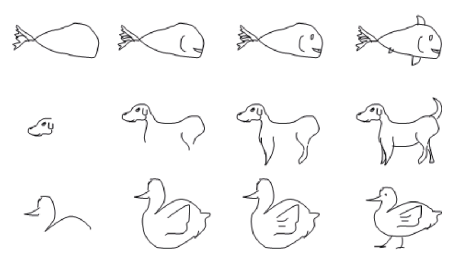

c. 草图绘制(4步)

图 2 草图绘制序列示例

\subsection{2 三维模型多视图序列的构建}

当前，针对三维模型的多视图构建算法较多， 如选择一张最佳视图的 ${ }^{[6]}$, 将三维模型正向摆放

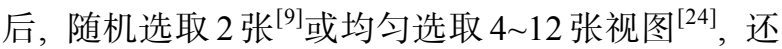
有围绕三维模型所在包围球随机采样选择 $k$ 个视 图 ${ }^{[11]}$. 鉴于真实世界中绝大多数物体本身就是正 向摆放的，人们观察物体时，也是在其周围多个视 角定点观察得出最终结论. 如图 3 所示, 本文选择 使用 MVCNN 算法 ${ }^{[24]}$, 将三维模型正向摆放, 然后 在其斜向上 $30^{\circ}$ 的位置，随机选取第 1 个视角，并以 此为起点, 均匀设置 12 个虚拟摄像机, 获得三维模 型 $v$ 所对应的多视图序列 $\wp(v)=\left(v_{1}, v_{2}, \cdots, v_{12}\right)$. 显 然，三维模型的多视图序列的图像顺序关系体现 了视图之间的位置关系.

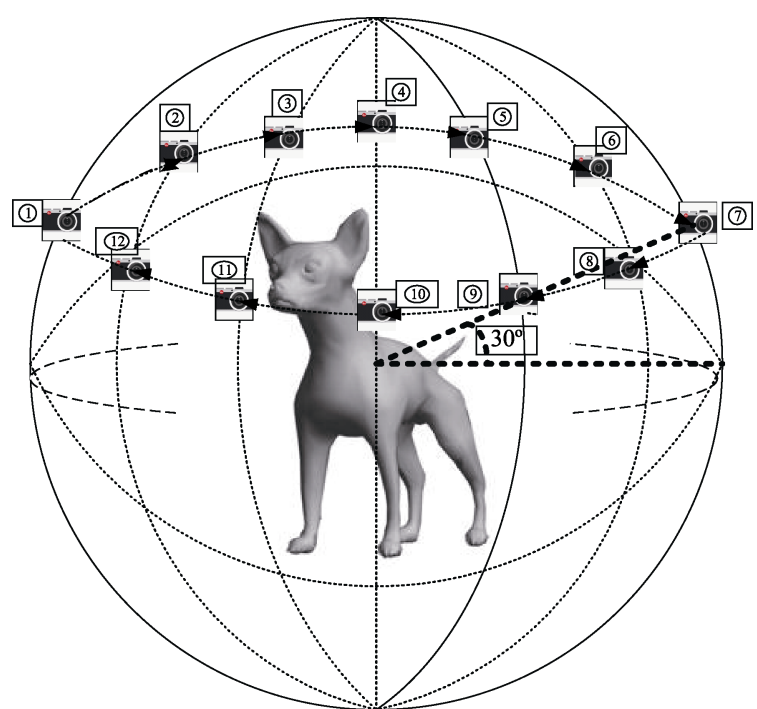

图 3 三维模型投影视图 


\section{2 联合时空特征分布的建立}

由第 2.1 节可知，草图绘制序列包括空域和时 域 2 部分信息: 空域以单个图像的形式存在, 体现 了绘制对象的形状; 时域以图像序列的形式存在, 体现了草图绘制的动态过程. 而在三维模型的多 视图序列中，单个视图体现了其空域信息，视图间 的顺序关系体现了一种观察视角连续变换的动态 信息 (可理解为时序信息). 本节旨在以草图绘制序 列和三维模型多视图序列为输人, 构建深度学习 网络，以有效地表征它们所包含的静态空域特征 和动态时序特征.

2017 年, 认知科学的研究者发现人脑认知视 频信息的神经网络分为 2 支：一支对应于腹侧流， 旨在提取视频的静态特征，即完成物体识别; 一支 对应于背侧流，旨在提取视频的动态特征，即完成 运动识别 ${ }^{[25]}$. 受此项研究结果启发, 本文拟模拟 人脑对视频的认知过程构建一种端到端的双流网 络, 如图 4 所示. 空间流网络 ResNet 旨在提取序列 中单个图像的静态空间特征，时间流网络 LSTM 旨在提取序列中所包含的动态时序特征, 两者串 行连接, 先提取静态特征, 再捕捉动态特征, 完成 动静信息的有效融合.

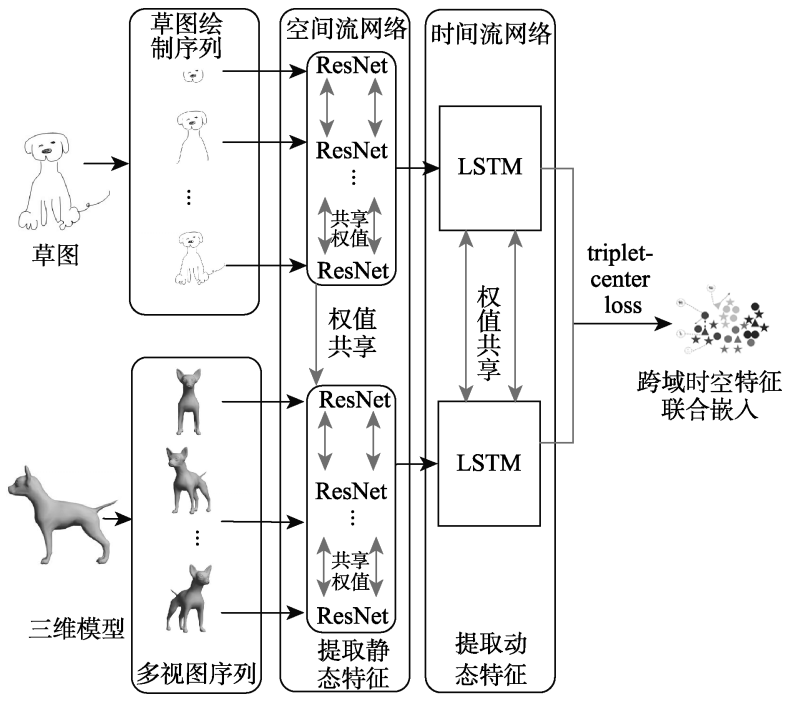

图 4 双流网络：联合时空特征分布的建立

\subsection{1 空间流网络：静态空间特征提取}

对于同一个模型，其所对应的草图数据和视 图数据拥有相同的内容属性和不同的风格属性, 这和跨域图像翻译中的跨域数据具有一致性. 白 静等 ${ }^{[13]}$ 根据跨域图像翻译原理，基于权值共享的 方式建立跨域数据联合特征映射，进而完成三维 模型草图检索, 并取得了很好的效果. 为此, 本文 将采用该方法通过权值共享的方式，实现草图和
视图数据静态特征的联合提取.

在网络选择方面, 针对图像序列中的每一幅 图像，本文将选择 CNN 提取其静态特征. 然而， 无论是草图还是三维模型对应的视图, 它们都不 是一般意义下的图像信息，没有丰富的纹理信息 和色彩信息. 草图数据线条较为稀疏, 总是利用较 长的轮廓线条体现被表征对象的外部形状; 视图 数据也总是包含一些体现三维模型整体结构的轮 廓信息和局部细节的几何信息. 为有效地捕获和 表征以上特征, 需要扩大感受野. 一些研究者提出 使用较大的卷积核扩大感受野，但是，大的卷积核 在扩大感受野的同时，也模糊了细节. 本文认为, 针对以上特点, 可在保持卷积核大小不变的情况 下，利用更深的网络来获取数据的大尺度信息，即 利用较深层次的网络获得更广的感受野, 做出更 加综合的判断.

理论分析表明, CNN 层次越深, 其网络的学习 能力就越强, 学习效果也越好. 但是, 在实践中, 针对普通的 $\mathrm{CNN}$, 当网络深度达到一定数目后, 继续叠加深度会造成网络难以训练, 出现梯度爆 炸和梯度消失等问题，导致训练精度和测试精度 迅速下降. 为此, 本文拟使用残差网络 ${ }^{[26]}$, 引人如 图 5 所示的短距离恒等映射, 在增加网络深度的同 时避免网络退化问题, 进而有效地提取草图和视图 中所包含的全局结构信息. 综合考虑图像规模、尺 寸和复杂度等因素，选用了残差网络 ResNet-50 $0^{[26]}$ 作为草图数据和视图数据静态特征的提取网络, 并通过在 ImageNet 上进行预训练, 提高网络的稳 定性，增加网络的收玫速度.

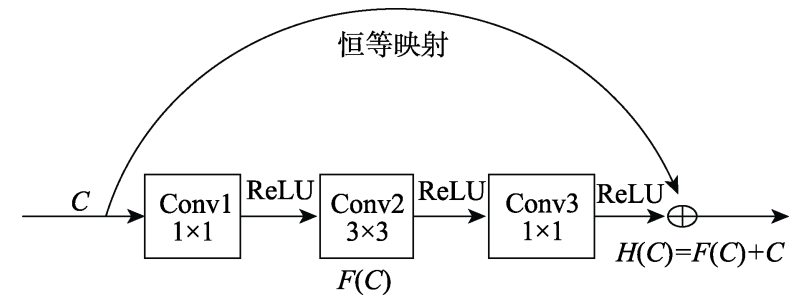

图 5 ResNet 残差结构

至此, 给定草图 $s$ 所对应的绘制序列 $\partial(s)$ 和三 维模型 $t$ 所对应的多视图序列 $\wp(v)=\left(v_{1}, v_{2}, \cdots, v_{12}\right)$ ， 可得草图静态特征序列 $f_{1}(\partial(s))=\left(f_{1}\left(s_{1}\right), \cdots, f_{1}\left(s_{m}\right)\right)$ 及 三维模型静态特征序列 $f_{2}(\wp(v))=f_{2}\left(\left(v_{1}\right), \cdots\right.$, $\left.f_{2}\left(v_{12}\right)\right)$.

2.2.2 构建时间流网络: 动态时序特征提取

由于草图的绘制序列有着一定的时序关系, 
与此同时，三维模型的视图之间也存在着位置相 关性，因此本文统一将其视为一种动态时序信息, 拟在得到静态特征之后构建时间流网络，对草图 和三维模型的动态特征进行提取, 进而得到动态 特征和静态特征相结合的特征描述.

综合比较各种表征时间序列的循环神经网络, 本文选择具有长短期记忆能力的网络 LSTM. 如图 6 所示, 将草图及三维模型的静态特征序列中第 $t$ 幅图像统一表征为 $x_{t}$, 则该网络通过 3 个不同的 门控单元(遗忘门、输人门、输出门)在长期记忆 $C_{t-1}$ 和当前输人信息 $x_{t}$ 之间进行选择和融合, 以捕获 具有长短期记忆能力的动态特征.

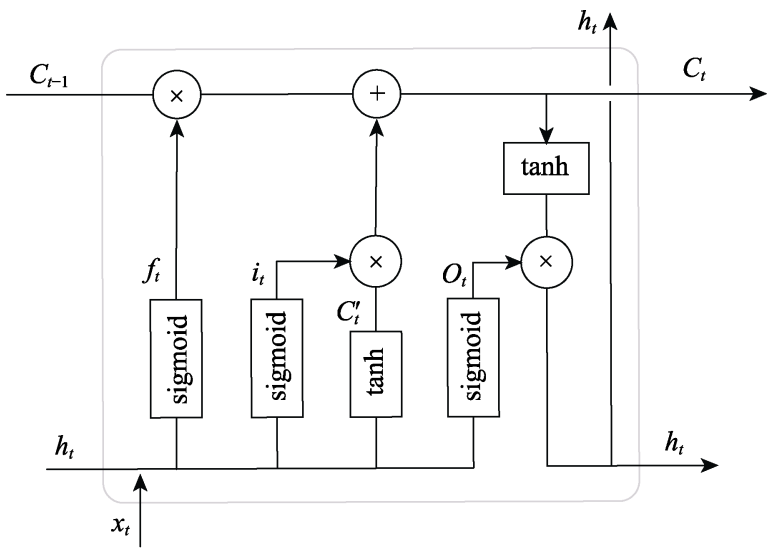

×逐点乘操作 丹逐点加操作

图 6 LSTM 网络单元

在图 6 中， $x_{t}$ 表示当前的输人， $h_{t-1}$ 表示上一 时刻的输出, $C_{t-1}$ 为上一时刻记忆单元的输出, $f_{t}$ 代表遗忘门， $i_{t}$ 代表输人门， $o_{t}$ 代表输出门， $S$ 代 表 sigmoid 函数, 具体计算公式为

$$
\left\{\begin{array}{l}
f_{t}=S\left(w_{f} \times\left[h_{t-1}, x_{t}\right]+b_{f}\right) \\
i_{t}=S\left(w_{i} \times\left[h_{t-1}, x_{t}\right]+b_{i}\right) \\
C_{t}^{\prime}=\tanh \left(w_{C} \times\left[h_{t-1}, x_{t}\right]+b_{C}\right) \\
C_{t}=f_{t} \times C_{t-1}+i_{t} \times C_{t}^{\prime} \\
o_{t}=S\left(w_{o} \times\left[h_{t-1}, x_{t}\right]+b_{o}\right) \\
h_{t}=o_{t} \times \tanh \left(C_{t}\right)
\end{array} .\right.
$$

其中, $w_{*}$ 和 $b_{*}$ 分别为对应网络的权重和偏置; sigmoid 函数输出 $0 \sim 1$ 的数值, 起到门控作用.

LSTM 单元的运行过程如下: 首先输人特征 $x_{t}$ 和上一时刻的输出 $h_{t-1}$, 经过神经元变换和激活 函数 $\tanh$ 作用后得到候选记忆单元 $C_{t}^{\prime}$, 捕捉当前 输人特征和短期记忆特征; 然后, 记忆单元的输出 $C_{t-1}$ 与遗忘门 $f_{t}$ 作用，决定多大程度的遗忘长期 记忆单元 $C_{t-1}$ 的信息; 候选记忆单元的输出 $C_{t}^{\prime}$ 与
输人门作用, 决定多大程度的保留输人特征和短 期记忆特征; 两者结合形成当前时刻的长期记忆输 出 $C_{t}$; 最后输出门 $o_{t}$ 与新的记忆单元 $C_{t}$ 决定信息 的输出 $h_{t}, h_{t}$ 和 $C_{t}$ 共同作用于下一个 LSTM 单元.

针对草图和三维模型的静态特征序列, 本文 将分别构建包含 $m$ 个和 12 个如图 6 所示网络单元 的 LSTM 网络, 以得到其最终特征向量 $\boldsymbol{R}(s)=$ $R\left(f_{1}(\partial(s))\right), \boldsymbol{R}(v)=R\left(f_{2}(\wp(v))\right)$.

\subsection{3 联合时空特征嵌人}

针对双流网络, 本节拟引人度量学习, 以完成 端到端的联合时空特征嵌人.

考虑草图绘制具有主观性，同类草图内部数 据之间可能存在较大差异, 难以满足二元度量学 习中“同类样本距离尽可能小”的严格距离约束, 本文拟引人三元度量学习算法, 放松要求, 确保类 内距离小于类间距离即可. 传统的 triplet loss 同时 考虑了类内距离和类间距离, 满足以上要求, 但是 其存在时间复杂度高、训练困难且费时的问题. 为 此, 本文采用 triplet-center loss ${ }^{[15]}$ 计算样本同类中 心之间的距离，在减少计算复杂度的同时，考虑类 内和类间距离.

为方便描述，本节将输人的草图和三维模型 统一表征为 $x^{i}$, 则其经过双流网络模型输出的特 征为 $R\left(x^{i}\right)$. 设该输人对应类标签为 $y_{i}$, 类内样本 的特征均值中心为 $C_{i}$, 其他类类内样本的特征均 值中心表征为 $C_{j}(j \neq i)$; 则输人样本 $x^{i}$ 与其类中 心的距离为 $D^{p}=D\left(R\left(x^{i}\right), C_{i}\right)$, 与其他类中心的距 离为 $D^{n}=D\left(R\left(x^{i}\right), C_{j}\right)$. 要求输人样本与同类样本 距离较近, 到其他类样本距离较远, 等价于输人样 本与同类样本中心之间的距离小于到其他类样本 中心的距离, 即 $\min D^{n}>D^{p}$; 引人决策边界 $\alpha(\alpha>0)$, 可进一步转化为 $\min D^{n}-D^{p}>\alpha$. 为此, 设定一个 batch 的样本数目为 $M$, 则 triplet-center loss 的损失函数可定义为

$$
L_{t-c}=\sum_{i=1}^{M} \max \left(\min _{j \neq i} D\left(R\left(x^{i}\right), C_{j}\right)-D\left(R\left(x^{i}\right), C_{i}\right)-\alpha, 0\right) .
$$

本文选用欧几里得度量作为特征之间的距离 度量函数, 即 $D\left(R\left(x^{i}\right), C_{j}\right)=\frac{1}{2}\left\|R\left(x^{i}\right)-C_{j}\right\|_{2}^{2}$.

为了在训练过程中更好地找到各个类的类中 心，建立更加鲁棒的损失函数，在 triplet-center loss 的基础上考虑分类损失，即加人 Softmax loss, 形成最终损失为 $L_{\text {total }}=w_{1} L_{t-c}+w_{2} L_{\text {Softmax }}$. 其中, 
$w_{1}$ 和 $w_{2}$ 为权重，旨在平衡度量损失和分类损失在 整个分类中所占的比例.

\section{3 实验结果}

\section{1 数据集}

为更好地和现有算法进行比较，本文选取被 广泛使用的 2 个大型公开数据集作为测试对象.

SHREC2013 $3^{[20-21]}$ 数据集包含 90 个类, 7200 张 草图和 1258 个三维模型. 各个类中的三维模型数 量不等，最少的只有 4 个，最多的则有 184 个; 各 个类中的草图数目均为 80 . 实验中, 针对每个类, 50 张草图用于训练, 30 张草图用于测试, 1258 个 三维模型则全部作为检索对象.

SHREC2014 $4^{[22-23]}$ 数据集规模更大，也更加复 杂, 共包含 171 个类, 13680 张草图和 8978 个三维 模型. 各个类中的三维模型数量最少的少于 10 个, 最多的则多于 300 个; 各个类中的草图数目也为 80. 实验中, 针对每个类, 50 张草图用于训练, 30 张草图用于测试, 8978 个三维模型全部作为检索 对象.

\section{2 评价指标}

本文使用检索的 7 个通用评价指标对实验结果 进行度量, 包括查全-查准率曲线 (precision-recall curves，PR)、最近邻(nearest neighbor，NN)、第 1 批次(first tier, FT)、第 2 批次 (second tier, ST), E 算 法(E-measure, E)、折扣累计收益(discounted cumulative gain, DCG)、平均准确率(mean average precision, MAP) ${ }^{[20-23]}$.

\section{3 实验设置}

\subsection{1 实验环境}

本文实验的硬件环境为 Intel Core i7 2600k + GTX 10808 GB+16 GB RAM, 软件环境为 Windows $10 \times 64+$ CUDA $\quad 9.0+\mathrm{CuDNN} \quad 7.1+$ PyTorch $1.3 .1+$ Python3.7+Matlab.

\subsection{2 数据处理及增强}

SHREC2013 和 SHREC2014 中提供的草图尺 寸为 $256 \times 256$ 像素. 相应地, 针对库内每个三维模 型, 本文采用 MVCNN 提供的多视图构建算法 ${ }^{[24]}$, 构建的视图尺寸也为 $256 \times 256$ 像素. 为了在静态 空间特征提取中使用在 ImageNet 数据集上预训练 好的网络模型, 在进行实验前, 针对每个草图和视 图, 采用随机裁剪、水平旋转、垂直旋转的方式增 强数据, 得到 $224 \times 224$ 像素的图像; 然后, 通过减 去图像内所有像素均值的方式对其进行归一化.
以上操作可以增加训练样本的数据量, 进而提高 模型的泛化能力, 在一定程度上避免网络过拟合, 提高预测的鲁棒性.

\subsection{3 实现细节}

基于增强后的每张草图, 本文选取 $m=3$, 构建 包含 3 个子草图的草图绘制序列; 针对每个三维模 型, 得到包含 12 个视图的视图序列. 然后, 选取在 ImageNet 数据集上做过预训练的 ResNet-50 作为 空间流网络，采用权值共享的方式，分别提取草图 及三维模型所对应的静态空间特征, 并将其转换 为 [bathzise，n，2 048] 的形式输人时间流网络 LSTM, 进一步提取草图和三维模型的动态时序特 征. 这里, $n$ 为三维模型视图数量和子草图序列数 目, 2048 为特征维度. 在训练过程中使用度量学 习 triplet-center loss 对网络训练的参数进行学习, 实现端到端学习的网络结构.

实验中, 网络的学习率为 $10^{-4}$, dropout 为 0.3 , 在 LSTM 中 hidden_size 为 2048, num_layers 为 2, 随机梯度下降(stochastic gradient descent, SGD)衰 减率为 0.9 ; 同时参照 triplet-center loss ${ }^{[15]}$ 的参数取 值，本文选取 $w_{1}=0.01 ， w_{2}=1.00$ ，阈值 $\alpha=5$; 检索 中采用欧几里得度量对特征进行相似度量.

\section{4 不同草图数的比较}

如图 3 所示, 草图绘制序列中, 不同个数的草 图包含的内容不同，体现的时序信息也不同. 为比 较不同草图个数对算法的影响, 在保证其他因素 一致的情况，本文依次采用 2 4 张子草图表征草图 动态绘制信息并完成检索，得到如表 1 所示的结 果. 由表 1 可见, 当草图数为 3 时, 算法得到了最 佳性能. 通过分析图 3 示例可以看到, 当草图数为 2 时, 体现的动态绘制信息过少, 因此算法性能较 差. 分析算法性能下降原因, 当草图数为 4 时, 尽 管草图绘制整体过程具有一致性，包含某种语义 信息, 但是单个笔画绘制中却存在主观性和差异 性. 当草图数目取值较大时, 相邻草图之间新增的 笔画数目较少, 就会更多地体现个别笔画绘制中 的差异性和主观性, 反而降低了算法的准确率. 在 后面的实验中, 草图数统一选取为 3 .

表 1 不同草图数的实验结果对比

\begin{tabular}{ccccccc}
\hline 草图数 & NN & FT & ST & E & DCG & MAP \\
\hline 2 & 0.850 & 0.805 & 0.845 & 0.407 & 0.910 & 0.844 \\
3 & $\mathbf{0 . 8 7 3}$ & $\mathbf{0 . 8 3 2}$ & $\mathbf{0 . 8 7 9}$ & $\mathbf{0 . 4 1 5}$ & $\mathbf{0 . 9 1 8}$ & $\mathbf{0 . 8 6 3}$ \\
4 & 0.851 & 0.802 & 0.850 & 0.409 & 0.912 & 0.857 \\
\hline
\end{tabular}

注. 加粗字体为最佳实验结果. 


\section{5 检索实验对比}

为充分测试算法有效性，在 SHREC2013 和 SHREC2014 数据集上的对比实验中, 本文选取了 7 种先进的三维模型草图检索算法与本文算法进 行综合对比. 具体地, 选取了 2 种传统的基于流行 排序的三维模型跨域草图检索 (cross-domain manifold ranking, CDMR)算法 ${ }^{[27]}$ 和基于视图聚类 和上下文匹配的三维模型草图检索(sketch-based 3D model retrieval based on view clustering and shape context matching, SBR-VC)算法 ${ }^{[28]}$; 选取了 5 种基于深度学习的算法: 基于深度相关度量学习的 DCML算法 ${ }^{[10]}$ 、基于二元度量学习的 Siamese算法 ${ }^{[8]}$ 、 基于 Wasserstein 质心表示(learned Wasserstein barycentric representation, LWBR)三维模型草图检索 算法 ${ }^{[9]}$ 、基于 triplet-center loss 构建静态联合特征 映射的 TCL 算法 ${ }^{[15]}$ 、基于深度共享语义空间嵌人 (deep common semantic space embedding, DCSSE) 的算法 ${ }^{[12]}$; 以及通过权值共享构建静态联合特征 映射(joint feature mapping, JFM)算法 ${ }^{[13]}$.

\subsubsection{SHREC2013 的检索结果及分析}

图 7 展示了各种算法在 SHREC2013 数据集上 的 PR 曲线(不包括 TCL 算法, 因为 TCL 算法未提 供查全率和查准率数据). (1) 本文算法在各个查全 率下均获得了最高的查准率, 整体性能优于当前 所有算法. (2) 与 PR 曲线整体表现最好 2 个算法 (JFM 和 DCSSE)相比较, 本文算法的平均准确率

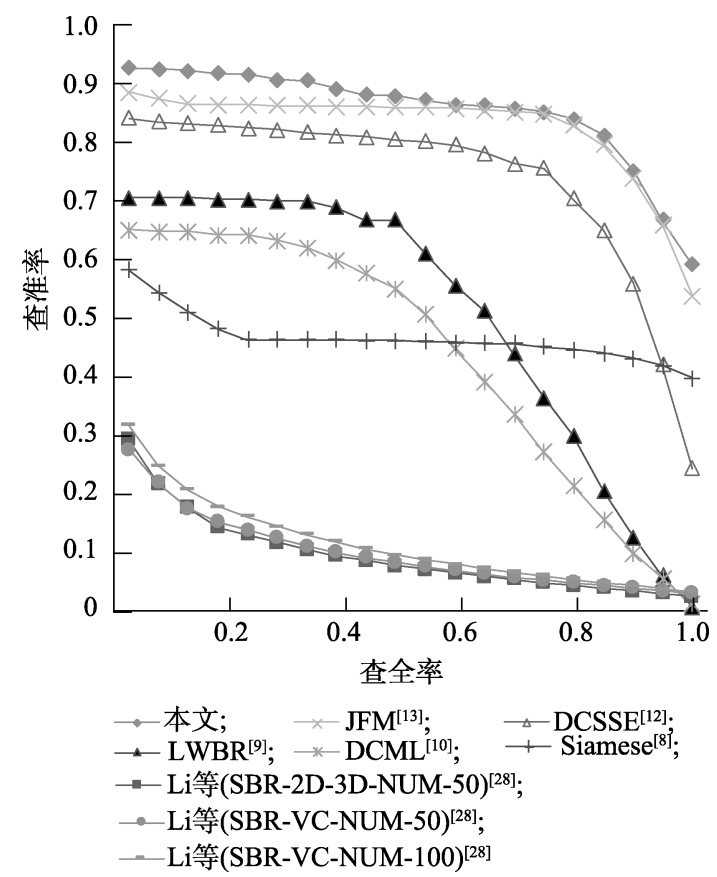

图 7 不同算法在 SHREC2013 数据集 PR 曲线对比
分别提高了 $4.5 \%$ 和 $4.6 \%$. (3) 与性能最接近的 JFM 算法比较，查全率低于 $50.0 \%$ 时，本文算法明显优 于 $\mathrm{JFM}$; 查全率高于 $50.0 \%$ 以后，两者查准率相当. 由于检索过程中往往更加关注最先返回的那部分 结果, 因此, 这一性能能够为用户提供更好的检索 感受. (4) 本文算法的检索性能非常稳定，在查全 率小于 $85.0 \%$ 时, 查准率在 $82.0 \%$ 以上; 在查全率 达到 $100 \%$ 时, 查准率仍然稳定在 $60.0 \%$. 以上数 据充分说明了本文算法的有效性. 由于 JFM 和 DCSSE 算法都采用了非常先进的手段对草图和三 维模型进行表征, 通过分析可知本文算法能够取 得更优的性能主要是因为在深度学习过程中引人 了草图和三维模型所对应的时序信息, 能够更加 全面地刻画被表征对象.

表 2 综合对比了各种算法在 SHREC2013 上的 检索指标. (1) 与传统算法及基于深度学习的经典 算法相比，本文算法在 6 项检索指标上都具有一定 的优势. (2) 与仅包含静态特征联合嵌人的 JFM 算 法 ${ }^{[13]}$ 相比，本文算法通过引人动态序列，检索性 能在 NN, FT, ST, E, DCG 和 MAP 这 6 项指标上分 别提高了 $2.5 \%, 5.7 \%, 1.7 \%, 0.6 \%, 2.8 \%$ 和 $4.5 \%$. 对 比结果充分验证了本文算法的有效性.

表 2 SHREC2013 数据集检索性能综合对比

\begin{tabular}{lcccccc}
\hline \multicolumn{1}{c}{ 算法 } & NN & FT & ST & E & DCG & MAP \\
\hline CDMR $^{[27]}$ & 0.279 & 0.203 & 0.296 & 0.166 & 0.458 & 0.250 \\
SBR-VC $^{[28]}$ & 0.164 & 0.097 & 0.149 & 0.085 & 0.348 & 0.116 \\
Siamese $^{[8]}$ & 0.405 & 0.403 & 0.548 & 0.287 & 0.607 & 0.469 \\
DCML $^{[10]}$ & 0.650 & 0.634 & 0.719 & 0.348 & 0.766 & 0.674 \\
LWBR $^{[9]}$ & 0.712 & 0.725 & 0.785 & 0.369 & 0.814 & 0.752 \\
TCL $^{[15]}$ & 0.763 & 0.787 & 0.849 & 0.392 & 0.854 & 0.807 \\
DCSSE $^{[12]}$ & 0.849 & 0.772 & 0.858 & 0.410 & 0.880 & 0.817 \\
JFM $^{[13]}$ & 0.848 & 0.775 & 0.862 & 0.409 & 0.890 & 0.818 \\
本文 & $\mathbf{0 . 8 7 3}$ & $\mathbf{0 . 8 3 2}$ & $\mathbf{0 . 8 7 9}$ & $\mathbf{0 . 4 1 5}$ & $\mathbf{0 . 9 1 8}$ & $\mathbf{0 . 8 6 3}$ \\
\hline
\end{tabular}

注. 加粗字体为最佳实验结果.

表 3 展示了 SHREC2013 中的部分检索结果, 左侧为随机选取的 7 个草图, 右侧为根据本文算法 获得前 10 个检索结果, 其中正确的模型着绿色, 错误的模型着橘黄色. 在 7 个检索实例中: (1) Airplane, Fish, Guitar, Tablelamp 这 4 个检索结果完全 正确; (2) Dog 的检索结果中前 7 个正确, 最后 3 个 错误, 通过查询数据集发现, 这是因为 Dog 类中仅 包含了 7 个三维模型, 在所有同类模型都已返回的 
表 3 SHREC2013 数据集部分检索实例展示

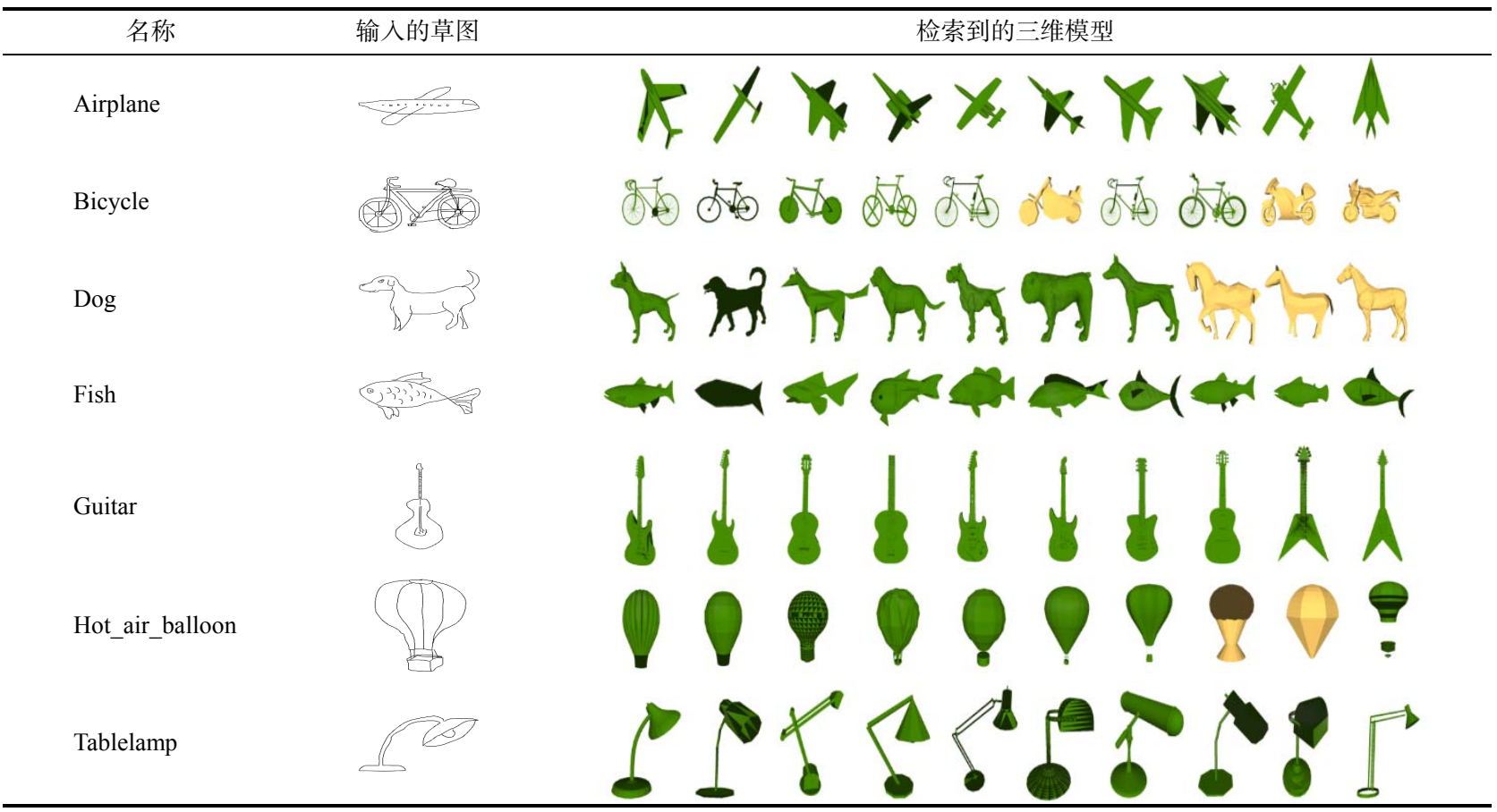

情况下, 算法根据相似度返回了最为接近的其他 3 个模型；(3) Bicycle 类也仅包含 7 个模型，返回的 10 个结果中已经完全包含了这 7 个模型, 只是由 于从形状来看, 第 7 个返回的摩托车较第 8 个结果 所示的自行车更接近草图, 因此产生了错误的排 序; (4) Hot_air_balloon 类内包含 9 个模型，返回的 前 10 个结果中包含了其中 8 个, 存在 2 个形状相 似的异类错误模型. 综合以上检索结果，本文算法 能够根据用户提供的草图较为准确、鲁棒地检索到 库内三维模型, 尽管存在少量错误, 但是都是形状 极为相似的难分模型.

\subsubsection{SHREC2014 的检索结果及分析}

为进一步测试本文算法在更复杂大型数据集上 的检索效果, 选取数据规模更大、类别更多、类内模 型数量差异更加突出的 SHREC2014 进行对比实验. 图 8 给出了各种算法在该数据集上的 PR 曲线, 表 4 所示为它们在 NN, FT, ST, E, DCG 和 MAP 这 6 项指 标上的对比结果. 由图 8 可以看出, 本文算法与现有 的多数算法相比依然保持较高的查全-查准率. 特别 值得注意的是, TCL 算法是一种使用 triplet-center loss 构建静态联合特征映射的检索算法 ${ }^{[15]}$, 本文是 使用 triplet-center loss 构建时空信息联合嵌入的检索 算法，而由表 4 的对比数据可知，本文算法在 SHREC2014 数据集上的各个检索指标都明显优于 TCL 算法, 并且在 5 项评价指标 FT, ST, E, DCG 和 MAP 上高于通过权值共享构建静态联合特征映射的 JFM 算法，再次验证了时空信息联合嵌人的有效性.

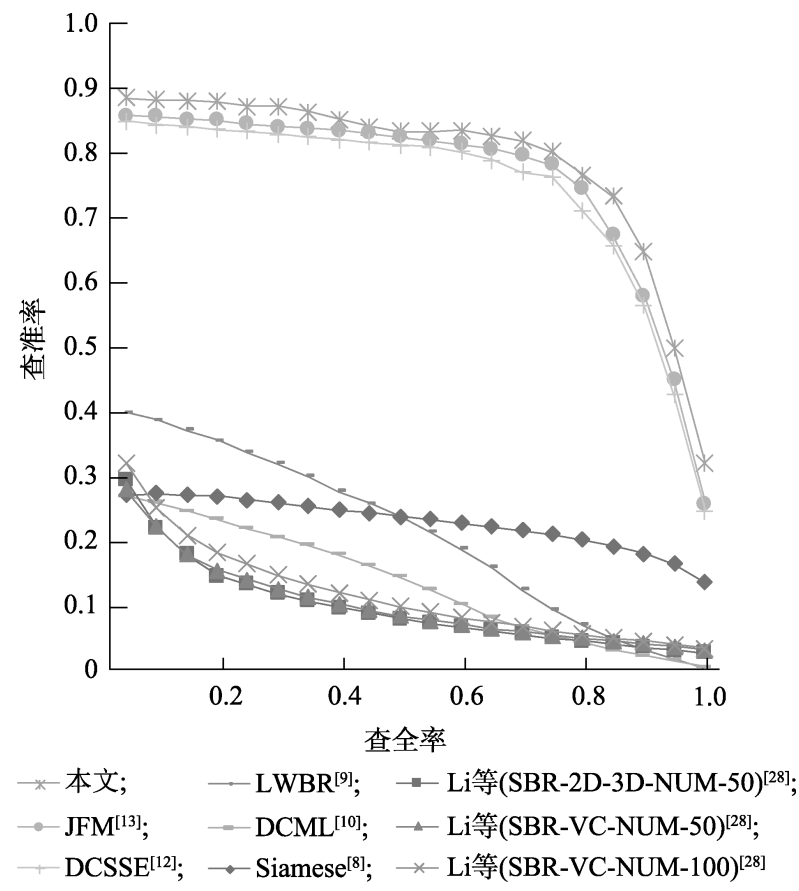

图 8 不同算法在 SHREC2014 数据集 PR 曲线对比

\section{6 讨 论}

通过对比表 2 和表 4 的数据可以明显发现，随 着数据集规模和复杂度的增加, 各个算法的检索 性能都有所下降. 表 5 给出了各个算法在 2 个数据 集上性能下降的比率. 同时明显发现一个规律，即 后 4 种算法的下降率和前 3 种算法的下降率大约相 差一个数量级. 通过分析可知, DCML 算法 ${ }^{[10]}$ 首先 利用传统的特征提取算法对三维模型进行表征, 
表 4 SHREC2014 数据集检索性能综合对比

\begin{tabular}{lcccccc}
\hline \multicolumn{1}{c}{ 算法 } & NN & FT & ST & E & DCG & MAP \\
\hline CDMR $^{[27]}$ & 0.109 & 0.057 & 0.089 & 0.041 & 0.328 & 0.054 \\
SBR-VC $^{[28]}$ & 0.095 & 0.050 & 0.081 & 0.037 & 0.319 & 0.050 \\
Siamese $^{[8]}$ & 0.239 & 0.212 & 0.316 & 0.140 & 0.496 & 0.228 \\
DCML $^{[10]}$ & 0.272 & 0.275 & 0.345 & 0.171 & 0.498 & 0.286 \\
LWBR $^{[9]}$ & 0.403 & 0.378 & 0.455 & 0.236 & 0.581 & 0.401 \\
TCL $^{[15]}$ & 0.585 & 0.455 & 0.539 & 0.275 & 0.666 & 0.477 \\
DCSSE $^{[12]}$ & 0.830 & 0.708 & 0.807 & 0.384 & 0.871 & 0.745 \\
JFM $^{[13]}$ & $\mathbf{0 . 8 3 5}$ & 0.720 & 0.810 & 0.390 & 0.873 & 0.756 \\
本文 & 0.817 & $\mathbf{0 . 7 2 2}$ & $\mathbf{0 . 8 2 0}$ & $\mathbf{0 . 4 0 1}$ & $\mathbf{0 . 8 7 3}$ & $\mathbf{0 . 7 8 7}$ \\
\hline
\end{tabular}

注. 加粗字体为对比最佳性能实验结果.

表 5 不同数据集检索性能下降比率

\begin{tabular}{lcccccc}
\hline \multicolumn{1}{c}{ 算法 } & NN & FT & ST & E & DCG & MAP \\
\hline DCML $^{[10]}$ & 0.49 & 0.57 & 0.53 & 0.50 & 0.35 & 0.59 \\
Siamese $^{[8]}$ & 0.41 & 0.47 & 0.42 & 0.51 & 0.18 & 0.51 \\
LWBR $^{[9]}$ & 0.43 & 0.48 & 0.42 & 0.36 & 0.36 & 0.47 \\
DCSSE $^{[12]}$ & 0.02 & 0.08 & 0.06 & 0.06 & 0.01 & 0.09 \\
JFM $^{[13]}$ & 0.02 & 0.07 & 0.06 & 0.05 & 0.02 & 0.08 \\
本文 & 0.06 & 0.13 & 0.06 & 0.03 & 0.05 & 0.09 \\
\hline
\end{tabular}

注. 下降比率算法为(SHREC2013 检索性能-SHREC2014 检索性能)/SHREC2013 检索性能.

然后利用深度学习完成跨模态数据的嵌人; Siamese ${ }^{[8]}$ 和 $\mathrm{LWBR}^{[9]}$ 这 2 种算法均在特征嵌人之前就 预先完成了三维模型多视图特征的融合. 以上算 法都可能在早期特征提取阶段或者特征融合阶段 造成有益信息的丢失，从而产生与整体最优解不 匹配的中间决策. 而 $\mathrm{DCSSE}^{[12]}, \mathrm{JFM}^{[13]}$ 以及本文算 法都是一种从原始输人到特征嵌人的端到端学习 网络，因此能够更好地形成整体最优解，也能够更 好地适应数据集规模和复杂度的变化. 这也从侧 面验证了端到端学习网络对跨模态检索问题的有 效性.

\section{4 结 语}

本文提出了一种基于时空信息联合嵌人的端 到端三维模型草图检索框架：将草图表征为动态 绘制序列, 将三维模型表征为多视图序列; 利用包 含静态空间特征提取的 ResNet 和包含动态时序特 征提取的 LSTM 构建双流网络, 实现草图和三维 模型时空特征的有效提取; 结合三元中心度量损 失建立草图和三维模型之间的跨域时空特征联合 嵌人，基于欧几里得度量完成草图-三维模型检索. 实验表明，本文通过联合捕获数据的静态空间特
征和动态时序特征, 能够更加充分、全面地表征跨 域检索对象所包含时空特征, 并进一步缩小跨域 数据之间的差异性, 取得了当前工作中较好的检 索性能. 未来考虑在当前的检索框架下引人注意 力机制, 以更好地关注显著特征, 进一步提高算法 在大规模数据集中的检索性能.

\section{参考文献(References):}

[1] Fang Y, Xie J, Dai G X, et al. 3D deep shape descriptor[C] // Proceedings of the IEEE Conference on Computer Vision and Pattern Recognition. Los Alamitos: IEEE Computer Society Press, 2015: 2319-2328

[2] Xie J, Fang Y, Zhu F, et al. DeepShape: deep learned shape descriptor for 3D shape matching and retrieval[C] //Proceedings of the IEEE Conference on Computer Vision and Pattern Recognition. Los Alamitos: IEEE Computer Society Press, 2015: 1275-1283

[3] Yoon S M, Scherer M, Schreck T, et al. Sketch-based 3D model retrieval using diffusion tensor fields of suggestive contours[C] //Proceedings of the 18th ACM International Conference on Multimedia. New York: ACM Press, 2010: 193-200

[4] Mao Dianhui, Yin Huanpu, Li Haisheng, et al. 3D model retrieval based on hand-drawn sketches[J]. Journal of System Simulation, 2015, 27(10): 2607-2614(in Chinese)

(毛典辉, 尹焕樸, 李海生, 等. 基于手绘草图的三维模型检 索[J]. 系统仿真学报, 2015, 27(10): 2607-2614)

[5] Fan Yachun, Tan Xiaohui, Zhou Mingquan, et al. A scale invariant local descriptor for sketch based 3D model retrieval[J]. Chinese Journal of Computers, 2017, 40(11): 2448-2465(in Chinese)

(樊亚春, 谭小慧, 周明全, 等. 基于局部多尺度的三维模型 草图检索方法[J]. 计算机学报, 2017, 40(11): 2448-2465)

[6] Liu Yujie, Song Yang, Li Zongmin, et al. Sketch-based 3D shape retrieval with representative view and convolutional neural network[J]. Journal of Graphics, 2018, 39(4): 735-741 (in Chinese)

(刘玉杰, 宋阳, 李宗民, 等. 融合信息熵和 $\mathrm{CNN}$ 的基于手 绘的三维模型检索[J]. 图学学报, 2018, 39(4): 735-741)

[7] Chen J X, Fang Y. Deep cross-modality adaptation via semantics preserving adversarial learning for sketch-based 3D shape retrieval[C] //Proceedings of the European Conference on Computer Vision. Heidelberg: Springer, 2018: 605-620

[8] Wang F, Kang L, Li Y. Sketch-based 3D shape retrieval using convolutional neural networks[C] //Proceedings of the IEEE Conference on Computer Vision and Pattern Recognition. Los Alamitos: IEEE Computer Society Press, 2015: 1875-1883

[9] Xie J, Dai G X, Zhu F, et al. Learning barycentric representations of 3D shapes for sketch-based 3D shape retrieval[C] // Proceedings of the IEEE Conference on Computer Vision and Pattern Recognition. Los Alamitos: IEEE Computer Society Press, 2017: 5068-5076

[10] Dai G X, Xie J, Fang Y, et al. Deep correlated holistic metric learning for sketch-based 3D shape retrieval[C] //Proceedings of the 31st AAAI Conference on Artificial Intelligence. Palo Alto: AAAI Press, 2017: 4002-4008 
[11] Chen J X, Qin J, Liu L, et al. Deep sketch-shape hashing with segmented 3D stochastic viewing[C] //Proceedings of the IEEE Conference on Computer Vision and Pattern Recognition. Los Alamitos: IEEE Computer Society Press, 2019: 791-800

[12] Bai J, Wang M J, Kong D X. Deep common semantic space embedding for sketch-based 3D model retrieval[J]. Entropy, 2019, 21(4): 369

[13] Bai Jing, Kong Dexin, Zhou Wenhui, et al. Joint feature mapping for end-to-end sketch-based 3D model retrieval [J]. Journal of Computer-Aided Design \& Computer Graphics, 2019, 31(12): 2056-2065(in Chinese)

(白静，孔德馨，周文惠，等. 基于联合特征映射的端到端三 维模型草图检索 $[\mathrm{J}]$. 计算机辅助设计与图形学学报, 2019, 31(12): 2056-2065)

[14] Eitz M, Hays J, Alexa M. How do humans sketch objects?[J]. ACM Transactions on Graphics, 2012, 31(4): Article No:44

[15] He X W, Zhou Y, Zhou Z C, et al. Triplet-center loss for multi-view 3D object retrieval[C] //Proceedings of the IEEE Conference on Computer Vision and Pattern Recognition. Los Alamitos: IEEE Computer Society Press, 2018: 1945-1954

[16] Zhao Peng, Liu Yang, Liu Huiting, et al. A sketch recognition method based on deep convolutional-recurrent neural network[J]. Journal of Computer-Aided Design \& Computer Graphics, 2018, 30(2): 217-224(in Chinese)

(赵鹏, 刘杨, 刘慧婷, 等. 基于深度卷积-递归神经网络的 手绘草图识别方法 $[\mathrm{J}]$. 计算机辅助设计与图形学学报, 2018, 30(2): 217-224)

[17] Li L, Zou C Q, Zheng Y Y, et al. Sketch-R2CNN: an $\mathrm{RNN}$-rasterization-CNN architecture for vector sketch recognition[J]. IEEE Transactions on Visualization and Computer Graphics, 2020, PP(99): 1

[18] Yu Q, Yang Y X, Song Y Z, et al. Sketch-a-net that beats humans[OL]. [2020-08-13]. https://arxiv.org/abs/1501.07873

[19] Xu P, Huang Y Y, Yuan T T, et al. SketchMate: deep hashing for million-scale human sketch retrieval[C] //Proceedings of the IEEE Conference on Computer Vision and Pattern Recognition.
Los Alamitos: IEEE Computer Society Press, 2018: 8090-8098

[20] Li B, Lu Y J, Godil A, et al. A comparison of methods for sketch-based 3D shape retrieval[J]. Computer Vision and Image Understanding, 2014, 119: 57-80

[21] Li B, Lu Y J, Godil A, et al. SHREC'13 track: large scale sketch-based 3D shape retrieval[C] //Proceedings of the 6 th Eurographics Workshop on 3D Object Retrieval. Aire-la-Ville: Eurographics Association Press, 2013: 89-96

[22] Li B, Lu Y J, Li C Y, et al. SHREC'14 track: extended large scale sketch-based 3D shape retrieval[C] //Proceedings of the 7th Eurographics Workshop on 3D Object Retrieval. Aire-la-Ville: Eurographics Association Press, 2014: 121-130

[23] Li B, Lu Y J, Li C Y, et al. A comparison of 3D shape retrieval methods based on a large-scale benchmark supporting multimodal queries[J]. Computer Vision and Image Understanding, 2015, 131: 1-27

[24] Su H, Maji S, Kalogerakis E, et al. Multi-view convolutional neural networks for 3D shape recognition[C] //Proceedings of the IEEE International Conference on Computer Vision. Los Alamitos: IEEE Computer Society Press, 2015: 945-953

[25] Zhou Aibao, Shen Sha, Jiang Qiang. Affordance: a perspective from mirror neurons $[\mathrm{J}]$. Journal of Psychological Science, 2017,40(6): 1335-1339(in Chinese)

(周爱保, 申莎, 蒋强. 示能性: 基于镜像神经元视角的理解 [J]. 心理科学, 2017, 40(6): 1335-1339)

[26] He K M, Zhang X Y, Ren S Q, et al. Deep residual learning for image recognition[C] //Proceedings of the IEEE Conference on Computer Vision and Pattern Recognition. Los Alamitos: IEEE Computer Society Press, 2016: 770-778

[27] Furuya T, Ohbuchi R. Ranking on cross-domain manifold for sketch-based 3D model retrieval[C] //Proceedings of the International Conference on Cyberworlds. Los Alamitos: IEEE Computer Society Press, 2013: 274-281

[28] Li B, Lu Y J, Godil A, et al. A comparison of methods for sketch-based 3D shape retrieval[J]. Computer Vision and Image Understanding, 2014, 119: 57-80 